\title{
The effectiveness of suicide prevention delivered by GPs: A systematic review and meta-analysis
}

\author{
Allison Milner ${ }^{\mathrm{a}, \mathrm{b}, *}$, Katrina Witt ${ }^{\mathrm{c}}$, Jane Pirkis ${ }^{\mathrm{d}}$, Sarah Hetrick ${ }^{\mathrm{e}}$,Jo Robinson ${ }^{\mathrm{e}}$, Dianne Currier ${ }^{\mathrm{d}}$, \\ Matthew J. Spittal ${ }^{\mathrm{d}}$, Andrew Page ${ }^{\mathrm{f}}$, Gregory L. Carter ${ }^{\mathrm{g}}$

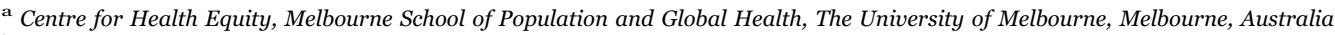 \\ b Work, Health and Wellbeing Unit, Population Health Research Centre, School of Health \& Social Development, Deakin University, Melbourne, Australia \\ c Turning Point, Monash University, Melbourne, Australia \\ d Centre for Mental Health, Melbourne School of Population and Global Health, The University of Melbourne, Melbourne, Australia \\ e Orygen National Centre of Excellence in Youth Mental Health, University of Melbourne, Melbourne, Australia \\ ${ }^{\mathrm{f}}$ Centre for Health Research, School of Medicine, Western Sydney University, Sydney, Australia \\ $\mathrm{g}$ Centre for Brain and Mental Health Research, Faculty of Health and Medicine, University of Newcastle, Newcastle, Australia
}

\section{A R T I C L E I N F O}

\section{Keywords:}

General practice

Suicide prevention

Self-harm

Suicide attempt

Suicide ideation

Doctor suicide prevention

\begin{abstract}
A B S T R A C T
Background: The aim of this review was to assess whether suicide prevention provided in the primary health care setting and delivered by GPs results in fewer suicide deaths, episodes of self-harm, attempts and lower frequency of thoughts about suicide.

Methods: We conducted a systematic review and meta-analysis using PRISMA guidelines. Eligible studies: 1) evaluated an intervention provided by GPs; 2) assessed suicide, self-harm, attempted suicide or suicide ideation as outcomes, and; 3) used a quasi-experimental observational or trial design. Study specific effect sizes were combined using the random effects meta-analysis, with effects transformed into relative risk (RR).

Results: We extracted data from 14 studies for quantitative meta-analysis. The RR for suicide death in quasiexperimental observational studies comparing an intervention region against another region acting as a "control" was 1.26 (95\% CI 0.58, 2.74). When suicide in the intervention region was compared before and after the GP program, the RR was 0.78 (95\% CI 0.62, 0.97). There was no evidence of a treatment effect for GP training on rates of suicide death in one cRCT (RR 1.07, 95\% CI 0.79, 1.45). There was no evidence of effect for the most other outcomes studied.

Limitations: All of the studies included in this review are likely to have a high level of bias. It is also possible that we excluded or missed relevant studies in our review process

Conclusions: Interventions have produced equivocal results, which varied by study design and outcome. Given these results, we cannot recommend the roll out of GP suicide prevention initiatives.
\end{abstract}

\section{Introduction}

Suicide and self-harm (including intentional self-injury or selfpoisoning irrespective of type of motivation and/or degree of suicidal intent) represents a serious public health burden. There is now good evidence that psychotherapeutic treatments (e.g., cognitive behaviour therapy or dialectical behavioural therapy) are effective at reducing the repetition of self-harm (Hawton et al., 2016). Results also suggest a non-significant reduction in suicide when using cognitive behavioural therapy and case management (Hawton et al., 2016).

However, a large number of individuals who are at risk of suicide may never come into contact with the specialist mental health services that offer these treatments (Appleby et al., 1999; Cavanagh et al., 2003; Law et al., 2010; Schaffer et al., 2016). In contrast, many people have contact with general practitioner (GP) services prior to suicide (Andersen et al., 2000; Leavey et al., 2016; Luoma et al., 2002; Pearson et al., 2009; Power et al., 1997; Stark et al., 2012). A review of over 40 studies (Luoma et al., 2002) found that up to three of four suicide victims had contact with primary care providers in the year of their suicide. More recently, a study from Northern Ireland found that as many as $85 \%$ of people who died by suicide were in contact with general practice services in the 12 months before their death (Leavey et al., 2016). In Scotland, $18.6 \%$ of those who died by suicide during the period 2001-2004 had contact with mental health services,

\footnotetext{
* Correspondence to: Centre for Health Equity, School of Population and Global Health, University of Melbourne, 207 Bouverie Street, Melbourne 3010.

E-mail address: Allison.milner@unimelb.edu.au (A. Milner).
} 


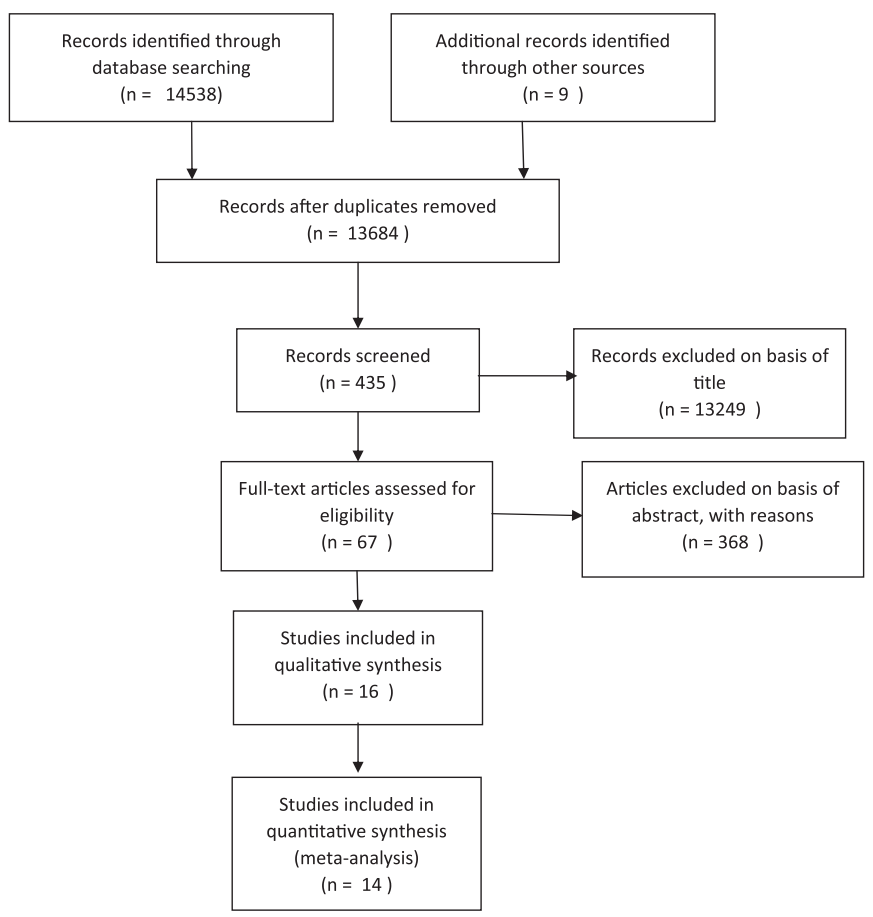

Fig. 1. PRISMA flow chart for study selection.

compared to $46.4 \%$ who had contact with general practice (Stark et al., 2012).

Given this evidence, it is unsurprising the involvement of GPs in providing suicide prevention services has been of considerable interest to researchers (Feltz-Cornelis et al., 2011; Leitner et al., 2008). There have also been several large-scale studies that feature GP training as a central component of suicide prevention initiatives (Hegerl et al., 2006, 2008; Roskar et al., 2010; Rutz et al., 1995, 1989a, 1992, 1990, 1989b, 1997). However, there has been limited assessment of the effectiveness of suicide prevention interventions that involve GPs. The aim of this review was to assess whether suicide prevention provided in the primary health care setting and delivered by GPs result in fewer suicide deaths, episodes of self-harm, attempts and thoughts about suicide.

\section{Methods}

The review was based on the Preferred Reporting Items for Systematic Reviews and Meta-Analyses (PRISMA) (Liberati et al., 2009).

\subsection{Search strategy and keywords}

We conducted a systematic search of seven electronic databases that index literature from a wide range of disciplines including intervention research (CENTRL-Trials Register), medical science (EMBASE; PubMED), public health (Global Health), psychology (PsycINFO), and social science (ProQuest; SCOPUS). We also searched for ongoing trials in the Australian and New Zealand Clinical Trials Register and the EU Clinical Trials Register. All databases were searched for eligible studies from their start date until 30 April 2016.

We used a three-tier search strategy to identify eligible studies. At the first stage, keywords related to general practitioners and general practice were combined (e.g., "general prac ${ }^{*}$ " OR "Primary Health Care"). At the second stage, these were combined with keywords inclusive of self-harm or suicide (e.g., self\$harm OR suicid* OR attempted suicid* OR parasuicid* OR "intentional\$self\$harm" OR "drug overdos*" OR auto\$mutilat* OR self\$cutt OR self $\$$ destructive behavio* OR self\$poison* OR self\$mutilat* OR self\$injur*). At the third stage, these were combined with keywords relating to suicide prevention or intervention (e.g., prevent* OR interven*). We originally also included keywords relating to psychoeducation (e.g., "health education" OR "health promotion" OR "medical education") in thirdtier searches but removed these after finding that the search produced a large number of non-relevant search results.

Keywords were adapted for the specific requirements of each electronic database. Truncation and wildcards were introduced where necessary to increase the sensitivity of the search. No restrictions were placed on publication status or language, but if we were unable to obtain adequate details for data extraction these studies were later excluded from meta-analyses.

Reference lists of identified studies, as well as prior relevant reviews in the field (Hawton et al., 2016; Lapierre et al., 2011; Mann et al., 2005; Roscoät and Beck, 2013; Roškar, 2012; Tait and Michail, 2014; van der Feltz-Cornelis et al., 2011; Zalsman et al., 2016) were hand screened to identify further relevant studies. Experts in the field were also contacted to assist with the identification of ongoing evaluations. Where necessary, corresponding authors were also contacted to clarify aspects of study design or methodology.

\subsection{Inclusion and Exclusion Criteria}

Studies were eligible for inclusion if: (1) they evaluated an intervention provided by GPs in primary care settings (i.e., the primary care provider was involved either solely or in combination with another support person in the delivery of the intervention), either as a standalone intervention or as part of a larger multicomponent intervention; (2) suicide, suicide attempt, self-harm or suicide ideation were assessed outcomes (whether primary or secondary), and; (3) they used a quasi-experimental observational (e.g., ecological before-after study, or an ecological study comparing a region that received the intervention against one that did no receive the intervention) or trial design, including cluster randomised controlled trials (cRCTs).

Studies were excluded if: (1) the intervention was not delivered, at least in part, by a GP (Unutzer et al., 2006 was excluded because the intervention was delivered by a Depression Care Manager rather than 


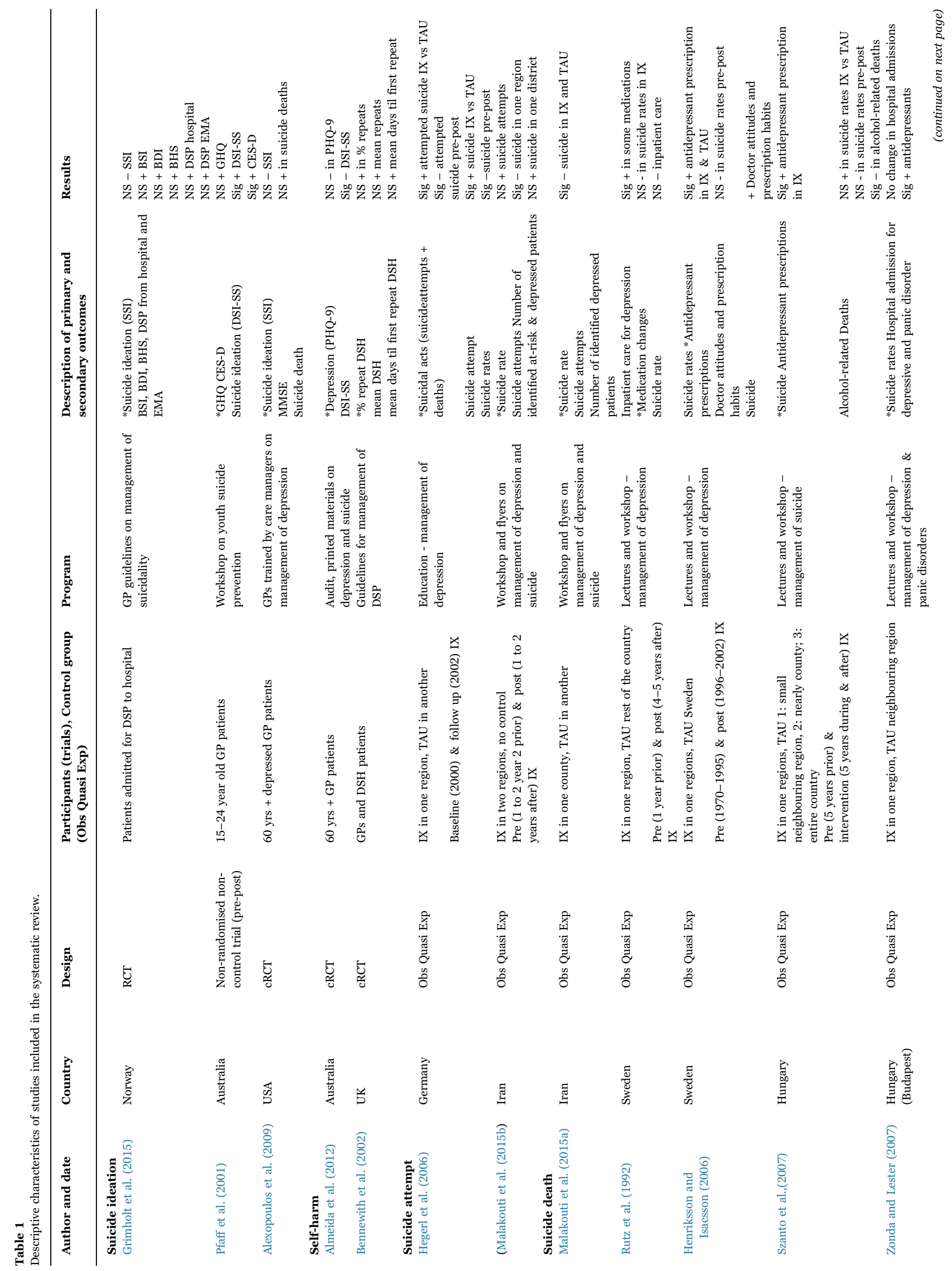


the GP); (2) the intervention comprised screening only, without an intervention. Study protocols and/or descriptions of programs not yet evaluated were also excluded (3).

Titles and abstracts of retrieved records were evaluated using a two stage screening process. At the first stage, studies with relevant titles were selected for second screening by one of the authors (AM). At the second stage, only those studies satisfying inclusion criteria following a review of the full-text were retained. Disagreements were resolved by consensus between two of the review authors (AM and KW).

\subsection{Statistical analysis}

For trials, including cRCTs, and quasi-experimental observational studies, we extracted data on number of individuals with and without subsequent episodes of suicidal ideation, self-harm, attempted and/or suicide death, where available. Given that cRCTs suffer from a substantial reduction in statistical efficiency as compared to standard RCTs, in which the individual is the unit of inference (Donner and Klar, 2004), we statistically adjusted for the effects of clustering following guidance outlined in the Cochrane Handbook (Higgins et al., 2008).

For quasi-experimental observational studies, in which data were reported as rates, rather than as proportions, these were extracted along with information on the total size of the population at risk during the relevant time period (a proxy for person-years). This enabled calculation of the study-specific incidence rate ratio and its $95 \%$ confidence intervals. Where data on population size was missing, but information on suicide rates per 100,000 persons and number of suicides were reported for each cohort, population size was estimated by dividing the number of suicides by the suicide rate, multiplied by 100,000 .

Data on the proportion of participants engaging in subsequent episodes of suicidal ideation, self-harm, attempted suicide, and/or suicide death were summarised for each study using the relative risk (RR) and 95\% confidence intervals. A RR of less than one indicates that the exposure (e.g., to the GP intervention) decreases the risk of an outcome (e.g., suicide) occurring; whereas an RR of greater than one indicates that the exposure increases the risk of that outcome occurring. An RR of equal to one indicates no associated between the exposure and the outcome of interest.

Study specific effect sizes were combined using the random effects meta-analysis to enable calculation of the pooled effect of interventions set in primary care on suicide ideation and behaviours (DerSimonian and Laird, 1986). Separate analyses were conducted for the outcomes of any suicide ideation, repeated self-harm, suicide attempts, and suicide death. We assessed the differences in treatment outcomes for women and men in studies where it was possible to do so (e.g., when these were proposed post hoc in subgroup analysis). Results were not pooled across different study designs due to considerable methodological differences in quasi-experimental observational versus trials. We transformed all effect measures into RRs.

Between-study heterogeneity was measured using the $I^{2}$ statistic, which indicates the percentage of between-study variability due to factors, such as participant or methodological differences, rather than chance alone (Higgins et al., 2003). By convention, $I^{2} \geq 75 \%$ represents substantial between-study heterogeneity (Higgins et al., 2003).

Analyses were undertaken in RevMan for Windows, version 5.3 (The Cochrane Collaboration, 2014).

The funders of the study had no role in study design, data collection, data analysis, data interpretation, or writing of the report. The corresponding author had full access to all the data in the study and had final responsibility for the decision to submit for publication.

\section{Results}

A total of 14,538 records were identified following the systematic search strategy outlined in Supplementary Document SD1. A further 


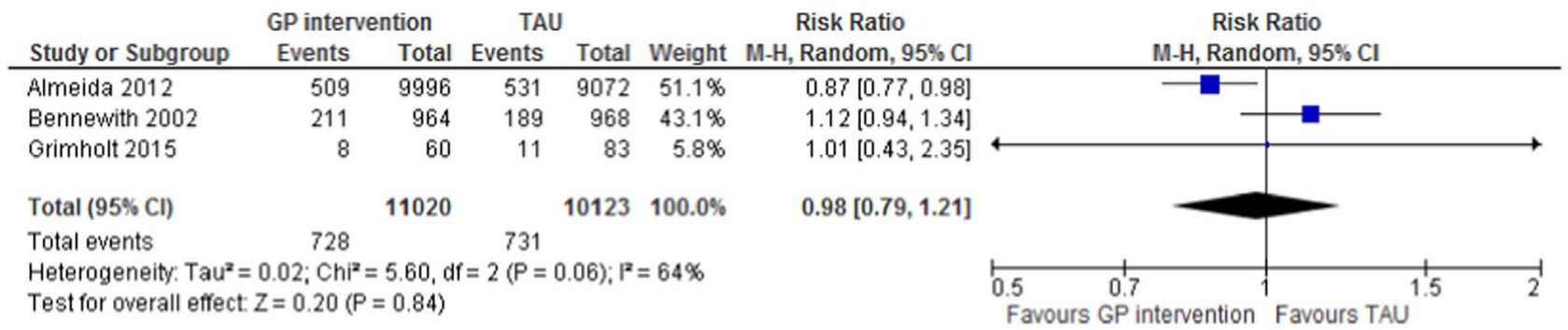

Fig. 2. Random effects odds ratio (OR) and 95\% confidence interval (CI) for repetition of self-harm.

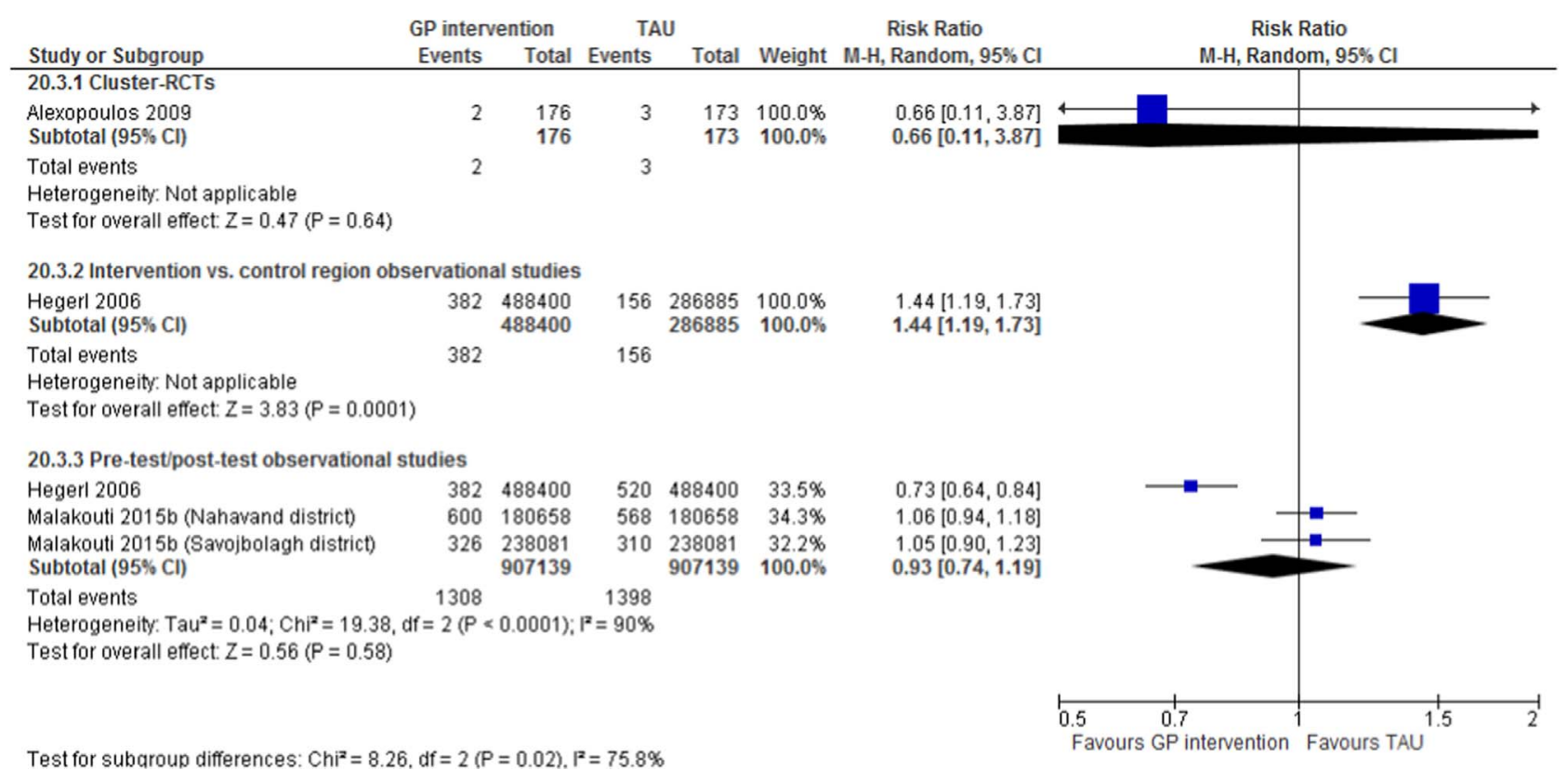

Fig. 3. Random effects odds ratio (OR) and $95 \%$ confidence interval (CI) for suicide attempts following GP training.

nine records were identified following snowballing and correspondence with researchers active in the field. Following deduplication, this was reduced to 13,684 . Of these, 13,249 were excluded at the first screening stage, and a further 368 were excluded following application of the inclusion and exclusion criteria at the second screening stage. A total of 16 records were therefore included in the present review comprising 16 independent, non-overlapping studies (Fig. 1). Of these, we extracted data from 14 studies for quantitative meta-analysis.

\subsection{Study characteristics}

The included studies were conducted in a variety of countries, such as Slovenia (Roskar et al., 2010), Sweden (Henriksson and Isacsson, 2006; Rutz et al., 1992), Japan (Oyama et al., 2006), Norway (Grimholt et al., 2015), Iran (Malakouti et al., 2015a, 2015b), Australia (Almeida et al., 2012; Pfaff et al., 2001) the UK (Bennewith et al., 2002; Morriss et al., 2005), the USA (Alexopoulos et al., 2009) Hungary (Szanto et al., 2007; Zonda and Lester, 2007) and Germany (Hegerl et al., 2008; Hübner-Liebermann et al., 2010).

Ten papers used a quasi-experimental observational design in which the efficacy of a GP intervention was assessed pre-post intervention as well as against a neighbouring control region (Hegerl et al., 2006; Henriksson and Isacsson, 2006; Hübner-Liebermann et al., 2010; Malakouti et al., 2015a, 2015b; Morriss et al., 2005; Oyama et al., 2006; Roskar et al., 2010; Rutz et al., 1992; Szanto et al., 2007; Zonda and Lester, 2007). These quasi-experimental observational studies often included GP training as one component of a multicomponent intervention strategy (Supplementary Document SD2). There were four RCTs or cRCTs (Alexopoulos et al., 2009; Almeida et al., 2012; Bennewith et al., 2002; Grimholt et al., 2015). One other trial did not randomise patients and had no control group (Pfaff et al., 2001).

Two studies reported outcomes on suicide ideation (Alexopoulos et al., 2009; Pfaff et al., 2001), three reported outcomes on self-harm (Almeida et al., 2012; Bennewith et al., 2002; Grimholt et al., 2015) or suicide attempt (Alexopoulos et al., 2009; Hegerl et al., 2006; Malakouti et al., 2015b) and ten reported outcomes on suicide deaths (Alexopoulos et al., 2009; Hegerl et al., 2006; Henriksson and Isacsson, 2006; Hübner-Liebermann et al., 2010; Malakouti et al., 2015a, 2015b; Oyama et al., 2006; Roskar et al., 2010; Rutz et al., 1992; Szanto et al., 2007).

More information on the study design characteristics can be seen in Table 1. Information on the content of the programs can be seen in Supplementary Document SD2.

\subsection{Meta-analysis}

Given the wide variation in study design used to assess the effectiveness of GP training (e.g., cRCTs, trial, and quasi-experimental observational designs), we included study design as a subgroup. We excluded two studies from the meta-analysis, as we were unable to extract the necessary data to include in a meta-analysis (Morriss et al., 


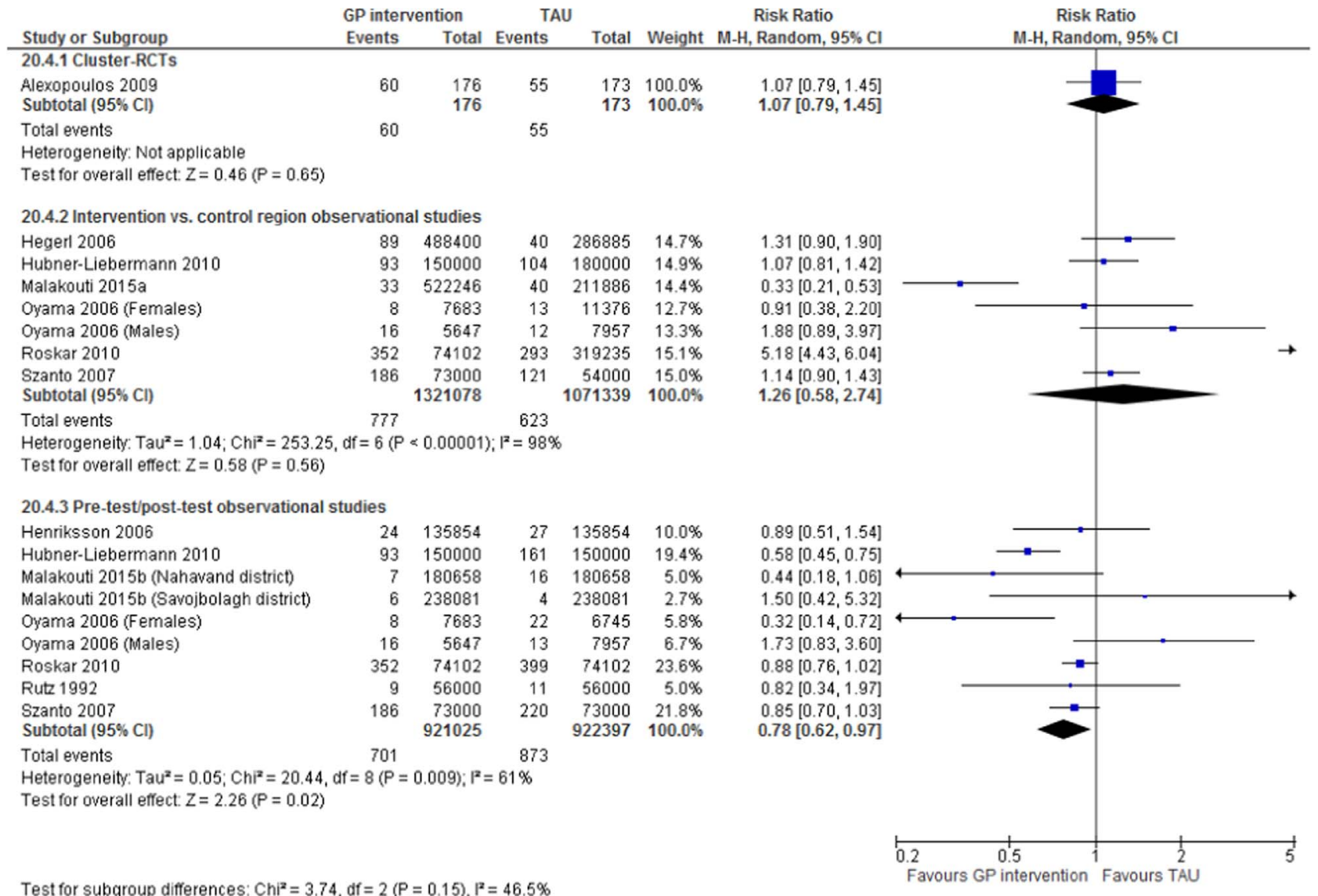

Fig. 4. Random effects odds ratio (OR) and 95\% confidence interval (CI) for suicides following GP training.

2005; Zonda and Lester, 2007).

As none of the cRCTs reported the information required to adjust for the effects of clustering (e.g., either the design effect or the intercluster coefficient) we could not adjust these analyses. These must therefore be interpreted with caution. For the quasi-experimental observational studies, in some it was possible to assess the effectiveness of the intervention compared with another region not receiving the intervention (which the authors argued operated as a control). It was possible in other quasi-experimental observational studies to assess differences prior to and following implementation of the intervention. Where possible, we assessed outcomes for both events.

\subsubsection{Suicidal ideation}

There was no evidence of a treatment effect for GP training on presentations for self-reported suicidal ideation in one cRCT [(Alexopoulos et al., 2009); intervention: 20/176 vs. TAU: 20/173; RR 0.98, 95\% CI 0.55-1.76; 1 study; $I^{2}=$ not applicable; $\left.p=0.95\right]$ or in one pre-test/post-test trial study [(Pfaff et al., 2001), 2001; Pre-test: 26/220 vs. Post-test: $21 / 220$; RR $1.24,95 \%$ CI $0.72-2.13 ; 1$ study; $I^{2}=$ not applicable; $p=0.44]$. There was no evidence of a difference in results by study design for this outcome $\left(\chi^{2}=0.32, d f=1, p=0.57\right)$. Heterogeneity was also not present for this outcome $\left(I^{2}=0 \%\right)$. But, as stated above, we decided not to pool results across study design due to concerns about methodological differences between study designs.

\subsubsection{Repetition of self-harm}

Three cRCTs investigated the effectiveness of GP training on repetition of self-harm (Almeida et al., 2012; Bennewith et al., 2002; Grimholt et al., 2015). There was no evidence of a treatment effect for GP training (Fig. 2). Heterogeneity was substantial $\left(I^{2=} 64.0 \%\right)$. However, as only three cRCTs were included in this analysis, we were unable to explore potential reasons for this heterogeneity.
Analysis by gender for one of these trials (Bennewith et al., 2002) suggested that whilst GP training may not lead to a reduction in selfharm repetition in males (intervention: $82 / 383$ vs. TAU: $84 / 413$; RR 1.05 , 95\% CI $0.80-1.38 ; 1$ study; $I^{2}=$ not applicable; $p=0.71$ ); this program may be effective in reducing self-harm repetition in females (intervention: $30 / 581$ vs. TAU: $105 / 555$; RR $0.27,95 \%$ CI $0.19-0.40$; 1 study; $I^{2}=$ not applicable; $\left.p<0.001\right)$.

\subsubsection{Suicide attempts}

GP training was not found to be associated with a significant treatment effect on suicide attempts in one cRCT (Alexopoulos et al., 2009) or in two pre-test/post-test quasi-experimental studies (Hegerl et al., 2006; Malakouti et al., 2015b). However, one quasi-experimental observational study comparing suicide reattempt rates in an intervention region as compared to a control region (Hegerl et al., 2006) suggested an increase in suicide attempts in the intervention region (Fig. 3), Sub-group analyses therefore suggested a significant difference in treatment effect by study design $\left(\chi^{2}=8.26, d f=2, p=0.02\right)$. Results also suggest substantial heterogeneity $\left(I^{2}=75.8 \%\right)$, thus justifying our decision to stratify by study design.

\subsubsection{Suicide death}

There was no evidence of a treatment effect for GP training on rates of suicide death in one cRCT (Alexopoulos et al., 2009), or in six quasiexperimental observational studies comparing suicide rates in an intervention region with a control region (Hegerl et al., 2006; Hübner-Liebermann et al., 2010; Malakouti et al., 2015a; Oyama et al., 2006; Roskar et al., 2010; Szanto et al., 2007). There was, however, evidence of a reduction in suicide rates in seven pre-test/ post-test quasi-experimental observational studies (Henriksson and Isacsson, 2006; Hübner-Liebermann et al., 2010; Malakouti et al., 2015b; Oyama et al., 2006; Roskar et al., 2010; Rutz et al., 1992; 
Szanto et al., 2007) (Fig. 4). There was no evidence of a difference by study design for this outcome, however $\left(\chi^{2}=3.74, d f=2, p=0.15\right)$. Between-study heterogeneity was also moderate for this outcome $\left(I^{2}=46.5 \%\right)$.

Post-hoc analysis on changes in suicide rates following implementation of a GP training program was available by gender for two intervention vs. control region quasi-experimental observational studies (Oyama et al., 2006; Roskar et al., 2010). There was no evidence of a treatment effect for GP training on suicide deaths for females (intervention: 79/37,051 vs. TAU: 78/37,051; RR 1.03, 95\% CI $0.75-1.40 ; 1$ study; $I^{2}=$ not applicable; $p=0.88$ ) in one study (Roskar et al., 2010). However, there was evidence to suggest that GP training may be associated with an increased rate of suicide in males (intervention: $273 / 37,051$ vs. TAU: $215 / 37,051$; RR 1.29 , 95\% CI $1.08-$ 1.54; 1 study; $I^{2}=$ not applicable; $p=0.006$ ) (Roskar et al., 2010).

Data obtained by correspondence for one study (Oyama et al., 2006) suggested that whilst the GP intervention was associated with a decrease in suicides in males at post-intervention in one region (Niigata Prefecture: IRR 0.84, 95\% CI 0.77-0.92), this effect was not observed for the intervention town itself (Matsudai: IRR 1.05, 95\% CI 0.51-2.19) (Oyama et al., 2006).

\subsubsection{Combined attempted and/or suicide deaths}

For one study, data on attempted suicide could not be disaggregated from data on suicide death (Hegerl et al., 2006). Comparing the intervention region with the control region in this study, there was some evidence to suggest GP training may be associated with a significant increase in attempted and/or suicide deaths (intervention: $471 / 488,400$ vs. control region $196 / 286,880$; RR 1.41 , 95\% CI $1.19-$ $1.67 ; 1$ study; $I^{2}=$ not applicable; $\left.p<0.001\right)$. However, suicide rates in the intervention region (i.e., Nuremberg) were significantly higher than in the control region (i.e., Würzberg) even at baseline. When comparing rates prior to the intervention to those following the intervention in Nuremberg only, therefore, GP training was associated with a significant decrease in attempted and/or suicide death in this study (Pretest: $620 / 488,400$ vs. Post-test: $471 / 488,400$; RR 0.76, 95\% CI $0.67-$ 0.86; 1 study; $I^{2}=$ not applicable; $p=<0.001$ ).

\section{Discussion}

This meta-analytic review focused on whether GP suicide prevention interventions (delivered either as a standalone intervention or as part of a larger multicomponent intervention) influenced suicide ideation, self-harm, suicide attempts, and suicide deaths. Below we present the limitations and the main results of the meta-analysis before discussing issues related to study design. We do this in order to encourage further debate about evaluation and effectiveness in suicide prevention.

\subsection{Limitations}

All of the studies included in this review are likely to have a high level of bias. Observational quasi-experimental studies were not randomised and were likely subject to the usual range of biases (especially selection bias). This raises the possibility that other factors (e.g., economic, social or geographical differences) might be responsible for any changes in suicidal behaviours in the intervention versus control region. Even in the RCTs, neither the GPs nor the participants were blinded to intervention condition, thus introducing the possibility of major biases, as those providing the intervention were not blind of intervention status. This non-blinding may also flow over to affect the analysis and interpretation of results. Other limitations include the possibility that we excluded or missed relevant studies in our review process. Finally, in a number of cases, there was only a small number of studies available for each meta-analysis, meaning that we weren't able to fully exploit the benefits of our analytic approach. Despite pooling samples across a number of studies, we will still underpowered to observe effects on suicide. We would also acknowledge that suicide was not a primary outcome in a number of studies (see Table 1) and that subgroup effects were proposed post-hoc. Last, it is important to highlight the substantial differences between the designs of the studies included in this review, which is why effect estimates were not pooled across study designs. In one case, this resulted in a relatively high $I^{2}$; in other cases, the $I^{2}$ was not applicable, as results could not be pooled across multiple studies. We limited our review to the assessment of GPs only, but acknowledge that there may be a range of other health professionals in primary care that could also be involved in providing suicide prevention services.

\subsection{Suicide deaths}

Most of the studies assessing suicide as an outcome were observational quasi-experimental studies. While there was evidence of moderate heterogeneity between studies for this outcome $\left(I^{2}=46.5 \%\right)$, the meta-analysis suggested that GP interventions were associated with a significant reduction in suicide rates using a pre-post evaluation design (using historical controls); however GP interventions were not associated with a significant effect when compared to a different region as a "control". This raises an interesting point about program evaluation in observational studies. One of the limitations of studies using this design is that they are unable to rule out the potential impact of external events that may influence suicide in an area (e.g., economic recession (Oyesanya et al., 2015)), and any changes cannot be attributed in a causal way. Further, intervention areas may have had a higher rate of suicide than controls prior to the intervention, thus affecting the decision to implement suicide prevention in these areas. There was only one cRCT that assessed suicide deaths finding that the GP intervention was associated with a non-significant increase in suicide deaths (Alexopoulos et al., 2009). However, suicide was only assessed outside the study period (five years later) in post hoc analyses. Given this, it is difficult to draw conclusions about the extent to which GP interventions are associated with suicide deaths in cRCT studies.

\subsection{Suicide attempts, self-harm, and suicide ideation}

A larger number of cRCTs assessed suicide ideation, attempts and self-harm (Alexopoulos et al., 2009; Almeida et al., 2012; Bennewith et al., 2002; Grimholt et al., 2015; Pfaff et al., 2001) compared to quasiexperimental studies (Henriksson and Isacsson, 2006; Malakouti et al., 2015a). Results suggest mixed effects, with some studies suggesting a beneficial effects and other suggesting a harmful effect. Similar to the studies evaluating suicide deaths, quasi-experimental observational studies found favourable effects when evaluations were pre-post using a historical control, but showed harmful outcomes when compared to regional controls. Two of the studies that assessed hospital treated selfharm found non-significant increases in presentations (Bennewith et al., 2002; Grimholt et al., 2015), while a third study found a reduction in self-reported self-harm (Almeida et al., 2012).

\subsection{Subgroup effects}

The cRCT by Bennewith et al. (2002) suggested subgroup effects, in that there was a decrease in self harm for females in the intervention groups, but not in males. Two quasi-experimental studies suggest an increase (Roskar et al., 2010) or no reduction (Oyama et al., 2006) in suicide rates for males. This raises the possibility that GP interventions for suicide are more effective for some groups than others. However, as subgroup effects were proposed post-hoc, we cannot over interpret the meaning of these results. 


\subsection{The suitability of GP interventions for other relevant outcomes}

Studies included in this review assessed a range of other outcomes related to suicide, including antidepressant use (Henriksson and Isacsson, 2006; Roskar et al., 2010), changes in GP knowledge (Pfaff et al., 2001), improvements in depressive symptomology (Alexopoulos et al., 2009; Almeida et al., 2012; Grimholt et al., 2015), referrals to outpatient and inpatient care (Rutz et al., 1992; Zonda and Lester, 2007), as well as selfharm and suicide attempts (Alexopoulos et al., 2009; Almeida et al., 2012; Bennewith et al., 2002; Grimholt et al., 2015; Pfaff et al., 2001). It is appropriate to assess these other outcomes as they are related to suicide, statistically common (and thus studies may have adequate ability to observe effects), and associated with help-seeking prior to suicide, which could be a legitimate aim/endpoint for interventions. However studies of this type need to articulate if and how changes in these outcomes are associated with suicidal behaviour.

\subsection{A comment on evaluation in GP interventions for suicide}

Many of the quasi-experimental observational studies identified in the current study were part of larger scale community interventions that included components of public education and awareness (hence, why we chose to analyse these separately from trial studies). The nature of these community interventions make it impossible to establish whether the GP intervention strategy alone was responsible for any changes in suicide, or whether there is an additional beneficial or harmful effect of the various intervention components. Regardless, these community level interventions are most appropriately assessed using an ecological approach (Craig et al., 2012), given that the units of analysis are by definition ecological variables. However, even when carefully designed, community-level interventions employing a 'comprehensive' approach to suicide prevention activity have important limitations, and assume additive effects when such approaches may be diluting potential effectiveness and finite resources over a range of targeted programs and services. Future studies should endeavour to assess how many of those who died by suicide post-intervention had contact with a GP prior to death. If GP interventions are effective in reducing suicide, then it would be expected that a lower rate of suicide would be evident among those who had contact with their GP compared to those who did not.

Another possible design that could be adopted in GP suicide prevention is the stepped wedge design, where the intervention is rolled-out sequentially and at random epochs of time to individual participants or clusters of individuals (Brown and Lilford, 2006). In stepped wedge designs, data collection occur at each point a new group (step) receives the intervention. These multiple data collection points would be difficult using self-reported methodologies as they constitute a high burden on individual participants, but are well suited to administrative forms of data collection (e.g., hospital records of selfharm or coronial suicide rates). As mentioned above, it may also be the case that trials are more suitably targeted towards statistically common factors related to suicide and self harm, while larger scale ecological studies may be better suited to assessing suicide as an outcome. Randomised trials and observational studies should aim to operate together to better evaluate impact on suicidal behaviours.

\section{Conclusion}

Interventions that target GP training for suicide prevention have produced equivocal results, which vary by study design and outcome. Considering the complexity of these interventions, we suggest that suicide prevention incorporating GP training carefully considers a multi-layered evaluation approach involving stepped wedge designs and cRCTs, as well as observational evaluation studies. Outcomes should be expanded to assess changes in exposure to risk factors for suicidal behaviours, as well as rates of suicidal behaviour.

\section{Appendix A. Supporting information}

Supplementary data associated with this article can be found in the online version at doi:10.1016/j.jad.2016.12.035.

\section{References}

Alexopoulos, G.S., Reynolds, C.F., Bruce, M., Katz, I., Raue, P.J., Mulsant, B.H., Oslin, D., Ten Have, T., 2009. Reducing suicidal ideation and depression in older primary care patients:patients 24-month: 24-month outcomes of the prospect study. Am. J. Psychiatry 166, 882-890.

Almeida, O.P., Pirkis, J., Kerse, N., Sim, M., Flicker, L., Snowdon, J., Draper, B., Byrne, G., Goldney, R., Lautenschlager, N.T., Stocks, N., Alfonso, H., Pfaff, J.J., 2012. A randomized trial to reduce the Prevalence of depression and self-harm behavior in older primary care patients. Ann. Fam. Med 10, 347-356.

Andersen, U.A., Andersen, M., Rosholm, J.U., Gram, L.F., 2000. Contacts to the health care system prior to suicide: a comprehensive analysis using registers for general and psychiatric hospital admissions, contacts to general practitioners and practising specialists and drug prescriptions. Acta Psychiatr. Scand. 102, 126-134.

Appleby, L., Shaw, J., Amos, T., McDonnell, R., Harris, C., McCann, K., Kiernan, K., Davies, S., Bickley, H., Parsons, R., 1999. Suicide within 12 months of contact with mental health services: national clinical survey. BMJ 318, 1235-1239.

Bennewith, O., Stocks, N., Gunnell, D., Peters, T.J., Evans, M.O., Sharp, D.J., 2002. General practice based intervention to prevent repeat episodes of deliberate self harm: cluster randomised controlled trial. BMJ 324, 1254.

Brown, C.A., Lilford, R.J., 2006. The stepped wedge trial design: a systematic review. BMC Med. Res. Methodol. 6, 1-9.

Cavanagh, J.T., Carson, A.J., Sharpe, M., Lawrie, S.M., 2003. Psychological autopsy studies of suicide: a systematic review. Psychol. Med. 33, 395-405.

Craig, P., Cooper, C., Gunnell, D., Haw, S., Lawson, K., Macintyre, S., Ogilvie, D. Petticrew, M., Reeves, B., Sutton, M., Thompson, S., 2012. Using natural experiments to evaluate population health interventions: new MRC guidance. J. Epidemiol. Community Health 66, 1182-1186.

DerSimonian, R., Laird, N., 1986. Meta-analysis in clinical trials. Control Clin. Trials 7, $177-188$.

Donner, A., Klar, N., 2004. Pitfalls of and controversies in cluster randomization trials. Am. J. Public Health 94, 416-422.

Feltz-Cornelis, C.M., v.d., Sarchiapone, M., Postuvan, V., Volker, D., Roskar, S., Grum, A.T., Carli, V., McDaid, D., O'Connor, R., Maxwell, M., Ibelshäuser, A., Audenhove, C.V., Scheerder, G., Sisask, M., Gusmão, R., Hegerl, U., 2011. Best practice elements of multilevel suicide prevention strategies. Crisis 32, 319-333.

Grimholt, T.K., Jacobsen, D., Haavet, O.R., Sandvik, L., Jorgensen, T., Norheim, A.B., Ekeberg, O., 2015. Effect of systematic follow-up by general practitioners after deliberate self-poisoning: a randomised controlled trial. PLoS One, 10.

Hawton, K., Witt, K.G., Taylor Salisbury, T.L., Arensman, E., Gunnell, D., Hazell, P., Townsend, E., van Heeringen, K., 2016. Psychosocial interventions for self-harm in adults. Cochrane Database Syst. Rev. Cd0, 12189.

Hegerl, U., Althaus, D., Schmidtke, A., Niklewski, G., 2006. The alliance against depression: 2-year evaluation of a community-based intervention to reduce suicidality. Psychol. Med. 36, 1225-1233.

Hegerl, U., Wittmann, M., Arensman, E., van Audenhove, C., Bouleau, J.H., van der Feltz-Cornelis, C., Gusmao, R., Kopp, M., Löhr, C., Maxwell, M., Meise, U., Mirjanic, M., Oskarsson, H., Sola, V.P., Pull, C., Pycha, R., Ricka, R., Tuulari, J., Värnik, A., Pfeiffer-Gerschel, T., 2008. The 'European alliance against depression (EAAD)': a multifaceted, community-based action programme against depression and suicidality. World J. Biol. Psychiatry 9, 51-58.

Henriksson, S., Isacsson, G., 2006. Increased antidepressant use and fewer suicides in Jamtland county, Sweden, after a primary care educational programme on the treatment of depression. Acta Psychiatr. Scand. 114, 159-167.

Higgins, J., Thompson, S., Deeks, J., Altman, D., 2003. Measuring inconsistency in metaanalysis. BMJ 327, 557-560.

Higgins, J.P.T., Deeks, J.J., Altman, D.G., on behalf of the Cochrane Statistical Methods Group, 2008. Special topics in statistics, In: Higgins, J.P.T., Green, S. (Eds.), Cochrane Handbook for Systematic Reviews of Interventions. Wiley \& Sons, Chichester, UK.

Hübner-Liebermann, B., Neuner, T., Hegerl, U., Hajak, G., Spießl, H., 2010. Reducing suicides through an alliance against depression? Gen. Hosp. Psychiatry 32, 514-518.

Lapierre, S., Erlangsen, A., Waern, M., de Leo, D., Oyama, H., Scocco, P., Gallo, J., Szanto, K., Conwell, Y., Draper, B., Quinnett, P., 2011. A systematic review of elderly suicide prevention programs. Crisis 32, 88-98.

Law, Y.-w., Wong, P.W., Yip, P.S., 2010. Suicide with psychiatric diagnosis and without utilization of psychiatric service. BMC Public Health 10, 1-11.

Leavey, G., Rosato, M., Galway, K., Hughes, L., Mallon, S., Rondon, J., 2016. Patterns and predictors of help-seeking contacts with health services and general practitioner detection of suicidality prior to suicide: a cohort analysis of suicides occurring over a two-year period. BMC Psychiatry 16, 1-8.

Leitner, M., Barr, W., Hobby, L., 2008. Effectiveness of inter- ventions to prevent suicide and suicidal behavior: A systematic review, Edinburgh, UK.

Liberati, A., Altman, D.G., Tetzlaff, J., Mulrow, C., Gøtzsche, P.C., Ioannidis, J.P.A., Clarke, M., Devereaux, P.J., Kleijnen, J., Moher, D., 2009. The PRISMA statement for reporting systematic reviews and meta-analyses of studies that evaluate healthcare interventions: explanation and elaboration. BMJ, 339.

Luoma, J.B., Pearson, J.L., Martin, C.E., 2002. Contact with Mental Health and Primary Care Providers Before Suicide: a Review of the Evidence. Am. J. Psychiatry 159, 
909-916.

Malakouti, S.K., Nojomi, M., Poshtmashadi, M., Hakim Shooshtari, M., Mansouri Moghadam, F., Rahimi-Movaghar, A., Afghah, S., Bolhari, J., Bazargan-Hejazi, S., 2015a. Integrating a suicide prevention program into the primary health care network: a field trial study in Iran. BioMed. Res. Int., 2015. http://dx.doi.org/ $10.1155 / 2015 / 193729$.

Malakouti, S.K., Nojomi, M., Poshtmashadi, M., Hakim Shooshtari, M., Mansouri Moghadam, F., Rahimi-Movaghar, A., Afghah, S., Bolhari, J., Bazargan-Hejazi, S. 2015b. Integrating of suicide prevention program into the primary health care network: a field clinical trial in Iran. Med J. Islam Repub. Iran. 29, 208.

Mann, J., Apter, A., Bertolote, J., et al., 2005. Suicide prevention strategies: a systematic review. JAMA 294, 2064-2074.

Morriss, R., Gask, L., Webb, R., Dixon, C., Appleby, L., 2005. The effects on suicide rates of an educational intervention for front-line health professionals with suicidal patients (the storm Project). Psychol. Med. 35, 957-960.

Oyama, H., Goto, M., Fujita, M., Shibuya, H., Sakashita, T., 2006. Preventing elderly suicide through primary care by community-based screening for depression in Rural Japan. Crisis 27, 58-65.

Oyesanya, M., Lopez-Morinigo, J., Dutta, R., 2015. Systematic review of suicide in economic recession. World J. Psychiatry 5, 243-254.

Pearson, A., Saini, P., Da Cruz, D., Miles, C., While, D., Swinson, N., Williams, A., Shaw, J., Appleby, L., Kapur, N., 2009. Primary care contact prior to suicide in individuals with mental illness. Br. J. Gen. Pr. 59, 825-832.

Pfaff, J.J., Acres, J.G., McKelvey, R.S., 2001. Training general practitioners to recognise and respond to psychological distress and suicidal ideation in young people. MJA $174,222-226$.

Power, K., Davies, C., Swanson, V., Gordon, D., Carter, H., 1997. Case-control study of GP attendance rates by suicides with or without a psychiatric history. Br. J. Gen. Pr. 47, 211-215.

Roscoät, Ed, Beck, F., 2013. Efficient interventions on suicide prevention: a literature review. Rev. D.'Épidémiologie Et. De. St. Publique 61, 363-374.

Roškar, S., 2012. Educational strategies for preventing suicide: review of studies in Slovenia, Gotland and Germany. Int. J. Psychiatry Clin. Pract. 16, 6.

Roskar, S., Podlesek, A., Zorko, M., Tavcar, R., Dernovsek, M.Z., Groleger, U., Mirjanic, M., Konec, N., Janet, E., Marusic, A., 2010. Effects of training program on recognition and management of depression and suicide risk evaluation for Slovenian primary-care physicians: follow-up study. Croat. Med. J. 51, 237-242.

Rutz, W., von Knorring, L., Pihlgren, H., Rihmer, Z., Wålinder, J., 1995. Prevention of male suicides: lessons from Gotland study. Lancet 345, 524.

Rutz, W., Von Knorring, L., Walinder, J., 1989a. Frequency of suicide on Gotland after systematic postgraduate education of general practitioners. Acta Psychiatr. Scan 80, $151-154$
Rutz, W., Von Knorring, L., Walinder, J., 1992. Long-term effects of an educational program for general practitioners given by the Swedish Committee for the prevention and treatment of depression. Acta Psychiatr. Scan 85, 83-88.

Rutz, W., von Knorring, L., Walinder, J., Wistedt, B., 1990. Effect of an educational program for general practitioners on Gotland on the pattern of prescription of psychotropic drugs. Acta Psychiatr. Scand. 82, 399-403.

Rutz, W., Walinder, J., Eberhard, G., Holmberg, G., von Knorring, A.L., von Knorring, L., Wistedt, B., Aberg-Wistedt, A., 1989b. An educational program on depressive disorders for general practitioners on Gotland: background and evaluation. Acta Psychiatr. Scand. 79, 19-26.

Rutz, W., Wålinder, J., Von Knorring, L., Rihmer, Z., Pihlgren, H., 1997. Prevention of depression and suicide by education and medication: impact on male suicidality. An update from the Gotland study. Int. J. Psychiatry Clin. Pr. 1, 39-46.

Schaffer, A., Sinyor, M., Kurdyak, P., Vigod, S., Sareen, J., Reis, C., Green, D., Bolton, J., Rhodes, A., Grigoriadis, S., Cairney, J., Cheung, A., 2016. Population-based analysis of health care contacts among suicide decedents: identifying opportunities for more targeted suicide prevention strategies. World Psychiatry 15, 135-145.

Stark, C.R., Vaughan, S., Huc, S., O'Neill, N., 2012. Service contacts prior to death in people dying by suicide in the Scottish Highlands. Rural Remote Health 12, 1876.

Szanto, K., Kalmar, S., Hendin, H., Rihmer, Z., Mann, J.J., 2007. A suicide prevention program in a region with a very high suicide rate. Arch. Gen. Psychiatry 64, 914-920.

Tait, L., Michail, M., 2014. Educational interventions for general practitioners to identify and manage depression as a suicide risk factor in young people: a systematic review and meta-analysis protocol. Syst. Rev. 3, 145.

The Cochrane Collaboration, 2014. Review Manager (RevMan) [Computer program]. Version 5.3. The Nordic Cochrane Centre, Copenhagen.

Unutzer, J., Tang, L., Oishi, S., Katon, W., Williams, J.W., Jr., Hunkeler, E., Hendrie, H., Lin, E.H.B., Levine, S., Grypma, L., 2006. Reducing suicidal ideation in depressed older primary care patients. J. Am. Geriatr. Soc. 54, 1550-1556.

van der Feltz-Cornelis, C.M., Sarchiapone, M., Postuvan, V., Volker, D., Roskar, S., Grum, A.T., Carli, V., McDaid, D., O'Connor, R., Maxwell, M., Ibelshäuser, A., Van Audenhove, C., Scheerder, G., Sisask, M., Gusmão, R., Hegerl, U., 2011. Best practice elements of multilevel suicide prevention strategies: a review of systematic reviews. Crisis 32, 319-333.

Zalsman, G., Hawton, K., Wasserman, D., van Heeringen, K., Arensman, E., Sarchiapone, M., Carli, V., Höschl, C., Barzilay, R., Balazs, J., Purebl, G., Kahn, J.P., Sáiz, P.A., Lipsicas, C.B., Bobes, J., Cozman, D., Hegerl, U., Zohar, J., 2016. Articles: suicide prevention strategies revisited: 10-year systematic review. Lancet Psychiatry 3 , 646-659.

Zonda, T., Lester, D., 2007. Preventing suicide by educating general practitioners. Omega 54, 53-57. 